\title{
A DUALITY IN INTEGRAL GEOMETRY; SOME GENERALIZATIONS OF THE RADON TRANSFORM ${ }^{1}$
}

\author{
SIGURDUR HELGASON
}

1. Introduction. It was proved by J. Radon in 1917 (see [26]) that a differentiable function $f$ of compact support on a Euclidean space can be determined from the integrals of $f$ over each hyperplane in the space. Whereas Radon was primarily concerned with the dimensions 2 and 3, the following formulation for an arbitrary Euclidean space $\boldsymbol{R}^{n}$ was given by John [23], [24]. If $\omega$ is a unit vector let $J(\omega, p)$ denote the integral of $f$ over the hyperplane $\left\{x \in R^{n} \mid\langle x, \omega\rangle=p\right\}$ where $\langle$,$\rangle denotes the inner product. Then, if d \omega$ denotes the surface element on the unit sphere $\Omega=S^{n-1}$, and $\Delta$ the Laplacian,

$$
\begin{array}{ll}
f(x)=\frac{1}{2}(2 \pi i)^{1-n} \Delta_{x}^{(n-1) / 2} \int_{\Omega} J(\omega,\langle\omega, x\rangle) d \omega & \text { (n odd), } \\
f(x)=(2 \pi i)^{-n} \Delta_{x}^{(n-2) / 2} \int_{\Omega} d \omega \int_{-\infty}^{\infty} \frac{d J(\omega, p)}{p-\langle\omega, x\rangle} \quad \text { ( } n \text { even), }
\end{array}
$$

where in the last integral, the Cauchy principal value is taken.

Applications. The applications of these formulas are primarily based on the following property: Consider the integrand in the integral over $\Omega$, say the function $J(\omega,\langle\omega, x\rangle)$ in (1). For a fixed $\omega$ this function $x \rightarrow J(\omega,\langle\omega, x\rangle)$ is a plane wave, that is a function which is constant on each member of a family of parallel hyperplanes. Aside from the Laplacian, formulas (1) and (2) give a continuous decomposition of $f$ into plane waves. Since a plane wave only amounts to a function of one real variable (along the normal to the hyperplanes) the formulas (1) and (2) can sometimes reduce a problem for $n$ real variables to a similar problem for one real variable. This principle has been used effectively on partial differential equations with constant coefficients (see Courant-Lax [2], Gelfand-Shapiro [10], John [24], Borovikov [1], Gårding [4]) and even for general elliptic equations (John [24]).

Generalizations. The above representation of a function by means of

An address delivered under the title The Radon transform on symmetric spaces before the Summer Meeting of the Society on August 30, 1963, in Boulder, Colorado by invitation of the Committee to Select Hour Speakers for Annual and Summer Meetings; received by the editors March 26, 1964.

${ }^{1}$ Work supported by the National Science Foundation, NSF GP-149. 
its plane integrals suggests the general question of determining a function $f$ on a space from the knowledge of the integrals of $f$ over certain subsets of the space. Radon himself discussed in [26] the problem of determining a function on the non-Euclidean plane from the integrals of the function over all geodesics, Funk [3] proved that a function $f$ on the 2 -sphere, symmetric with respect to the center, can be determined by means of the integrals of $f$ over the great circles. The theory of representations of noncompact semisimple Lie groups, in particular the Plancherel formula, raises the problem of determining a function on a semisimple Lie group by means of its integrals over all conjugacy classes and their translates (see Gelfand-Naimark [9], Harish-Chandra [12], [14], Gelfand-Graev [6]). Other examples can be found in Gelfand [5], Gelfand-Graev [7], Gelfand-GraevVilenkin [8], Hachaturov [11], Harish-Chandra [13], Helgason [16], [19], [21], John [24], Kirillov [25] and Semyanistyi [30].

Notation. If $M$ is any manifold, $\varepsilon(M)$ denotes the space of $C^{\infty}$ functions on $M, D(M)$ the space of $C^{\infty}$ functions on $M$ with compact support. If $M$ is a complete Riemannian manifold, $s(M)$ denotes the space of rapidly decreasing functions on $M$ as defined in [21]. We recall that $f \in \delta(M)$ if and only if the following condition is satisfied. Let $\Delta_{1}, \cdots, \Delta_{n}$ be the Laplace-Beltrami operators of the various factors in the local de Rham decomposition of $M$ into irreducible parts [27]. Each $\Delta_{i}$ can be viewed as a differential operator on $M$. Then $f \in \mathcal{S}(M)$ if and only if $P\left(\Delta_{1}, \cdots, \Delta_{n}\right) f$ goes to zero at $\infty$ faster than any power of the distance from a fixed point.

2. Dual integral transforms. As mentioned above, formulas (1) and (2) give a continuous decomposition of $f$ into plane waves. We shall now focus attention on a kind of a projective duality which appears in (1). Formula (1) contains two integrations, dual to each other: first one integrates over the set of points in a given hyperplane, then one integrates over the set of hyperplanes passing through a given point. Guided by this duality we adopt the following general setup.

(i) Let $X$ be a manifold and $G$ a transitive Lie transformation group of $X$. Let $\Xi$ be a family of subsets of $X$, permuted transitively by $G$; in particular, there is induced a $G$-invariant differentiable structure on $\Xi$. The manifold $\Xi$ will be called the dual of $X$. Let $\boldsymbol{D}(X)$ and $\boldsymbol{D}(\boldsymbol{\Xi})$ denote the algebras of $G$-invariant differential operators on $X$ and $\Xi$, respectively.

(ii) Given $x \in X$, let $\check{x}=\{\xi \in \Xi \mid x \in \xi\}$. It is assumed that each $\xi$ and each $\check{x}$ have measures, say $\mu$ and $\nu$, respectively, such that the 
action of $G$ on $X$ and $\Xi$ permutes the measures $\mu$ and permutes the measures $\nu$.

(iii) If $f$ and $g$ are suitably restricted functions on $X$ and $\Xi$, respectively, we can define functions $\hat{f}$ on $\Xi$, $\check{g}$ on $X$, by

$$
\hat{f}(\xi)=\int_{\xi} f(x) d \mu(x), \quad \check{g}(x)=\int_{\check{x}} g(\xi) d \nu(\xi) .
$$

We shall now discuss several examples within this framework. The problems which will be considered are:

A. Relate function spaces on $X$ and $\Xi$ by means of the integral transforms $f \rightarrow \hat{f}$ and $g \rightarrow \check{g}$.

B. Does there exist a map $D \rightarrow \hat{D}$ of $D(X)$ into $D(\Xi)$ and a map $E \rightarrow \check{E}$ of $D(\Xi)$ into $D(X)$ such that

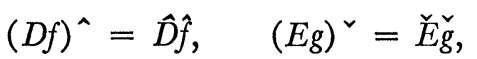

for general $f$ and $g$ ?

C. In case the transforms $f \rightarrow \hat{f}$ and $g \rightarrow \breve{g}$ are one-to-one, find explicit inversion formulas. In particular, find the relationships between $f$ and $(\hat{f})^{\vee}$ and between $g$ and $(\check{g})^{\wedge}$.

3. Points and hyperplanes in a Euclidean space. Let $X=\boldsymbol{R}^{n}(n>1)$ and let $G$ be the group of all rigid motions of $X$ and $\Xi$ the set of all hyperplanes in $X$. If $x \in X, \xi \in \Xi$ the measure $\mu$ on $\xi$ is the ordinary Euclidean measure and the measure $\nu$ on $x$ is the unique measure on the compact set $\check{x}$, invariant under all rotations around $x$, normalized by $\nu(\check{x})=1$. A hyperplane $\xi \in \Xi$ is determined by a unit normal vector $\omega$ and a real number $p$ such that $p \omega \in \xi$. The pairs $(\omega, p)$ and $(-\omega,-p)$ give the same $\xi$, the manifold $S^{n-1} \times R$ is a double covering of $\Xi$ and $C^{\infty}$ functions on $\Xi$ will be identified with $C^{\infty}$ functions $F$ on $S^{n-1} \times R$ satisfying $F(\omega, p)=F(-\omega,-p)$. Accordingly, let $\delta(\Xi)$ denote the set of rapidly decreasing functions $F$ on the Riemannian manifold $\boldsymbol{S}^{n-1} \times R$, satisfying $F(\omega, r)=F(-\omega,-r)$. Similarly $D(\Xi)$ and $\varepsilon(\Xi)$ are defined. Let $S_{H}(\Xi)$ denote the set of $F \in S(\Xi)$ such that for each integer $k \geqq 0$, the integral $\int_{-\infty}^{\infty} F(\omega, p) p^{k} d p$ can be written as a homogeneous $k$ th degree polynomial in $\omega_{1}, \cdots, \omega_{n}$. Let $D_{H}(\Xi)$ $=\mathfrak{D}(\Xi) \cap S_{H}(\Xi)$. Finally, let $\mathcal{S}^{*}(X)=\mathcal{S}^{*}\left(\boldsymbol{R}^{n}\right)$ denote the set of $f \in \mathcal{S}(X)$ satisfying $\int f(x) p(x) d x=0$ for each polynomial $p$ and $s^{*}(\Xi)$ the set of functions $F \in \mathcal{S}(\boldsymbol{\Xi})$ satisfying $\int_{-\infty}^{\infty} F(\omega, p) p^{k} d p=0$ for all $\omega$ and all integers $k \geqq 0$.

TheOREM 3.1. The algebra $D(X)$ is generated by the Laplacian $\Delta$, and the algebra $D(\Xi)$ is generated by the differential operator $\square: g(\omega, p)$ $\rightarrow d^{2} / d p^{2}(g(\omega, p))$. Moreover 


$$
(\Delta f)^{\wedge}=\square \hat{f}, \quad(\square g)^{\vee}=\Delta \check{g}
$$

for $f \in \mathcal{S}(X), g \in \mathcal{E}(\Xi)$.

THEOREM 3.2. The Radon transform $f \rightarrow \hat{f}$ is a linear one-to-one mapping of $\mathfrak{D}(X)$ onto $\mathfrak{D}_{H}(\Xi)$, of $\mathfrak{S}(X)$ onto $\mathcal{S}_{H}\left(\Xi^{\prime}\right)$, and of $\mathrm{S}^{*}(X)$ onto $\mathrm{S}^{*}\left(\Xi^{\prime}\right)$.

Theorem 3.1 is proved in [21]. The first statement of Theorem 3.2 holds in the following sharper form [21]: If $f \in \mathcal{S}(X)$ and if $\hat{f}(\xi)=0$ for all $\xi$ at distance $>R$ from 0 then $f(x)$ vanishes identically for $|x|>R$. The second part of Theorem 3.2 is, in a different form, stated in Gelfand-Graev-Vilenkin [8]. The proof given there (pp. 34-39) seems incomplete in some respects; for example, it uses (1) $\$ 1$ and therefore leaves out the even-dimensional case. A different proof is given in [21] using the relation

$$
\tilde{f}(r \omega)=\int_{-\infty}^{\infty} \hat{f}(\omega, p) e^{-i p r} d p
$$

connecting the Radon transform $\hat{f}$ and the Fourier transform $\tilde{f}$ of $f$. The last part of Theorem 3.2 is stated in Semyanistyi [29].

THEOREM 3.3. The following inversion formulas hold.

If $n$ is odd,

$$
\begin{aligned}
& f=c \Delta^{(n-1) / 2}\left((\hat{f})^{\vee}\right), \\
& f \in \mathcal{S}(X) \text {; } \\
& g=c \square^{(n-1) / 2}\left((\check{g})^{\wedge}\right), \\
& g \in \mathcal{S}^{*}(\Xi)
\end{aligned}
$$

where the constant $c$ is given by

$$
c=\Gamma\left(\frac{n}{2}\right)^{-1}(2 \pi i)^{1-n} \pi^{n / 2} .
$$

If $n$ is even,

$$
\begin{array}{ll}
f=c_{1} J_{1}\left((\hat{f})^{\vee}\right), & f \in \mathcal{S}(X) ; \\
g=c_{2} J_{2}\left((\breve{g})^{\wedge}\right), & g \in \mathcal{S}^{*}(\Xi),
\end{array}
$$

where $J_{1}$ and $J_{2}$ are given by analytic continuation ${ }^{2}$

$$
\begin{aligned}
& J_{1}: \quad f(x) \rightarrow \underset{\substack{\alpha=1-2 n \\
\text { anal. cont. }}}{\int_{R^{n}}} f(y)|x-y|^{\alpha} d y, \\
& J_{2}: F(\omega, p) \rightarrow \underset{\beta=-n}{\operatorname{anal.cont}} \int_{R} F(\omega, q)|p-q|^{\beta} d q,
\end{aligned}
$$

and

${ }^{2}$ In the notation of Schwartz [28, p. 45], $J_{1} f=\left(P f \cdot r^{1-2 n}\right) * f, J_{2} F=\left(P f \cdot r^{-n}\right) * F$. 


$$
c_{1}=\frac{\pi^{1-n} \Gamma\left(n-\frac{1}{2}\right)}{\Gamma\left(\frac{n}{2}\right) \Gamma\left(\frac{1}{2}-\frac{n}{2}\right)}, \quad c_{2}=\frac{\pi^{(1-n) / 2}}{\Gamma\left(\frac{1}{2}(1-n)\right)}
$$

The odd-dimensional case in Theorem 3.3 is a direct consequence of (1) $\$ 1$ and Theorem 3.1. The inversion formula (3) can be derived from (2) $\$ 1$ by means of some computation involving the distribution $P f \cdot r^{1-2 n}$ (see [21]). The formula for $g$ then follows by use of the Fourier transform.

4. Points and antipodal manifolds in compact two-point homogeneous spaces. Let $X$ be a compact two-point homogeneous space, that is, a compact Riemannian manifold with the property that the group $G$ of all isometries of $X$ acts transitively on the set $\{(x, y) \in X$ $\times X \mid d(x, y)=r\}$ for each fixed $r>0$. Here $d$ denotes distance. Let $L$ denote the diameter of $X$. If $x \in X$, let $A_{x}$ denote the corresponding antipodal manifold, that is, the set of points $y \in X$ at distance $L$ from $x$. This $A_{x}$ is a totally geodesic submanifold of $X$ and with the Riemannian structure on $A_{x}$ induced by that of $X, A_{x}$ is another twopoint homogeneous space. Also $x \neq y \Rightarrow A_{x} \neq A_{y}$. Let $\Xi$ denote the set of antipodal manifolds, with the differentiable structure induced by the transitive action of $G$. The Lie group $G$ is a compact semisimple Lie group (ignoring the trivial case $\operatorname{dim} X=1$ ). Changing the Riemannian structure on $X$ by a constant factor we may assume it induced by the negative of the Killing form of the Lie algebra of $G$. On $\Xi$ we choose the Riemannian structure such that the diffeomorphism $\phi: x \rightarrow A_{x}$ is an isometry. Let $\Delta$ and $\hat{\Delta}$ denote the LaplaceBeltrami operators on $X$ and $\Xi$, respectively. The measures $\mu$ and $\nu$ on the manifolds $\xi$ and $\check{x}$ will be those induced by the Riemannian structures of $X$ and $\Xi$. If $x \in X$, then $\check{x}=\{\phi(y) \mid y \in \phi(x)\}$. Consequently

$$
\check{g}(x)=\int_{\check{x}} g(\xi) d \nu(\xi)=\int_{y \in \phi(x)} g(\phi(y)) d \nu(\phi(y))=\int_{\phi(x)}(g \circ \phi)(y) d \mu(y)
$$

so

$$
\check{g}=(g \circ \phi)^{\wedge} \circ \phi .
$$

Because of this correspondence between the integral transforms $f \rightarrow \hat{f}$ and $g \rightarrow \check{g}$ it suffices to consider the first.

Concerning Problems A, B and C the following theorem holds. 
TheOREM 4.1. The mapping $f \rightarrow \hat{f}$ is a linear one-to-one mapping of $\mathcal{E}(X)$ onto $\mathcal{E}(\Xi)$ and

$$
(\Delta f)^{\wedge}=\hat{\Delta} \hat{f} \text {. }
$$

Except for the case when $X$ is an even-dimensional real projective space,

$$
f=P(\Delta)(\hat{f})^{2}, \quad f \in \mathcal{E}(X),
$$

where $P$ is a polynomial, independent of $f$, explicitly given below. The algebras $D(X)$ and $D(\Xi)$ are generated by $\Delta$ and $\hat{\Delta}$, respectively.

This theorem is proved in [21] by an elaboration of the method in $[16$, p. 284], where the case of odd-dimensional projective spaces is settled. The general case requires Wang's classification [31] of compact two-point homogeneous spaces as the following spaces: The spheres $S^{n}(n=1,2, \cdots)$, the real projective spaces $P^{n}(R)$ $(n=2,3, \cdots)$, the complex projective spaces $P^{n}(C)(n=4,6, \cdots)$, the quaternion projective spaces $P^{n}(H)(n=8,12, \cdots)$, and the Cayley projective plane $P^{16}(\mathrm{Cay})$. The superscript denotes the real dimension. The antipodal manifolds in the respective cases are a point, $P^{n-1}(R), P^{n-2}(C), P^{n-4}(H), S^{8}$. The polynomial $P(\Delta)$ above has degree equal to one half the dimension of the antipodal manifold, and is a constant multiple of

1 (the identity) $\quad X=S^{n}$

$$
\begin{array}{cl}
(\Delta-\kappa(n-2) 1)(\Delta-\kappa(n-4) 3) \cdots(\Delta-\kappa 1(n-2)) & X=P^{n}(R) \\
(\Delta-\kappa(n-2) 2)(\Delta-\kappa(n-4) 4) \cdots(\Delta-\kappa 2(n-2)) & X=P^{n}(C) \\
{[(\Delta-\kappa(n-2) 4)(\Delta-\kappa(n-4) 6) \cdots(\Delta-\kappa 8(n-6))]} & \\
\cdot[(\Delta-\kappa 4(n-4))(\Delta-\kappa 4(n-2))] & X=P^{n}(H) \\
(\Delta-112 \kappa)^{2}(\Delta-120 \kappa)^{2} & X=P^{16}(\text { Cay }) .
\end{array}
$$

In each case $\kappa=(\pi /(2 L))^{2}$, where $L$ is the diameter of $X$.

In the exceptional case when $X$ is an even-dimensional real projective space there is still an inversion formula $f=K\left((\hat{f})^{2}\right)$ but now $K$ is an integral operator. Considering functions on $X$ as symmetric functions on $S^{n}, K$ is given by a suitably regularized integral operator

$$
(K \phi)(x)=c \int_{S^{n}} F\left(\frac{n}{2}, \frac{n}{2}, \frac{1}{2} ; \sin ^{2}(d(x, y))\right) \phi(y) d \omega(y),
$$

where $d \omega$ is the volume element of $\boldsymbol{S}^{n}, c$ is a constant and $F$ is the hypergeometric function (Semyanistyi [30]).

REMARK. There is an important difference in the duality above between points and antipodal manifolds and in the duality between 
points and hyperplanes in $\S 3$. In the first case we have a diffeomorphism of $X$ onto the manifold $\Xi$ of antipodal manifolds. In the second the polarity with respect to the unit sphere $|x|=1$ gives a one-to-one mapping of $X-\{0\}$ onto the subset of $\Xi$ consisting of those hyperplanes which do not pass through 0 . This special role of 0 is the reason underlying the appearance of the spaces $\mathscr{D}_{H}(\boldsymbol{\Xi}), \varsigma_{H}(\Xi)$ in Theorem 3.2 in place of the spaces $D(\Xi)$ and $s(\Xi)$.

5. Points and totally geodesic hypersurfaces in a hyperbolic space. Let $n$ be an integer $>1$ and consider the quadratic form

$$
Q(Z) \equiv z_{1}^{2}+\cdots+z_{n}^{2}-z_{n+1}^{2}, \quad Z=\left(z_{1}, \cdots, z_{n+1}\right),
$$

on the Euclidean space $R^{n+1}$. Let $X$ denote the quadric $Q(Z)+1=0$ with the Riemannian structure induced by $Q$. Let $G$ be the group $O(n, 1)$ of linear transformations of $R^{n+1}$ leaving $Q$ invariant. The Riemannian manifold $X$ has constant curvature -1 and $G$ is a transitive Lie group of isometries of $X$. The totally geodesic submanifolds of $X$ of dimension $n-1$ are obtained as intersections of $X$ with hyperplanes in $R^{n+1}$ through the origin. A normal (with respect to $Q$ ) to such a hyperplane intersects the quadric $Q(X)-1=0$ in two points so the set of totally geodesic hypersurfaces-the dual space $\Xi$-can be identified with the quadric $Q(X)-1=0$ with symmetric points identified. This identification is consistent with the action of $G$. The quadratic form $Q$ induces a pseudo-Riemannian structure of signature $(n-1,1)$ and constant curvature +1 on $\Xi$ (see $[17$, p. 146]). In group-theoretic terms we have

$$
X=O(n, 1) / O(n), \quad \Xi=O(n, 1) /\left(O(n-1,1) \times Z_{2}\right),
$$

where $\boldsymbol{O}(n)$ is the orthogonal group in $\boldsymbol{R}^{n}$ and $\boldsymbol{Z}_{2}$ is the group with two elements. Since the group $G$ acts isometrically, $\mu$ and $\nu$ can be taken as the measures induced by the pseudo-Riemannian structures of $X$ and $\Xi$; concerning $\nu$ one has to remark that for $x \in X$ the pseudoRiemannian structure of $\Xi$ is nondegenerate on the submanifold $\check{x}$ of $\Xi$.

Concerning Problems A, B and C we have the following results.

Theorem 5.1. The algebras $\boldsymbol{D}(X)$ and $\boldsymbol{D}(\Xi)$ are generated by the Laplace-Beltrami operators $\Delta$ and $\Delta$ on $X$ and $\Xi$, respectively, and

$$
(\Delta f)^{\wedge}=\hat{\Delta} \hat{f} \text { for } f \in D(M) .
$$

If $n$ is odd,

$$
f=c(\Delta+1(n-2))(\Delta+3(n-4)) \cdots(\Delta+(n-2) 1)(\hat{f})^{\vee}
$$


where $c$ is a constant, independent of $f$.

The last formula is proved in [16]. An inversion formula for the even-dimensional case, more complicated to state, can be found in [30].

For the space $X$ the volume of a ball of radius $r$ increases with $r$ like $e^{(n-1) r}$. This explains the growth condition in the next result. Let $o$ be a fixed point in $X$; if $x \in X, \xi \in \Xi$ then $d(o, x)$ and $d(o, \xi)$ denote the corresponding distances from $o$.

Theorem 5.2. Suppose $f \in \mathcal{E}(X)$ satisfies

(i) For each integer $m>0, f(x) e^{\operatorname{md}(o, x)}$ is bounded;

(ii) $\hat{f}(\xi)=0$ if $d(o, \xi)>R$.

Then

$$
f(x)=0 \text { for } d(o, x)>R .
$$

6. Points and horocycles in a symmetric space [19]. Let $X$ be a symmetric space of the noncompact type [18] and $G$ the largest connected group of isometries of $X$ in the compact open topology. The group $G$ has center reduced to the identity element and thus can be identified with its adjoint group. This matrix group contains maximal unipotent subgroups, all known to be mutually conjugate, and their orbits in $X$ are called horocycles. The group $G$ acts transitively on the set $\Xi$ of all horocycles. The transform $f \rightarrow \hat{f}$ is defined by integration over each horocycle $\xi$, the volume element being that induced by the Riemannian structure of $X$. The transform $g \rightarrow g$ is defined by averaging over each $\check{x}, x \in X$, the compact isotropy subgroup of $G$ at $x$ acting transitively on $\check{x}$.

Let $G=K A N$ be an Iwasawa decomposition of $G$ where the subgroups $K, A, N$ are compact, abelian and unipotent, respectively. Let $M$ denote the centralizer of $A$ in $K$. Then we have the following identifications from the natural action of $G$ on $X$ and $\Xi$,

$$
X=G / K, \quad \Xi=G / M N .
$$

The manifolds $X$ and $\Xi$ have the same dimension. The polar coordinate decomposition of Euclidean space, i.e. the mapping $(\omega, p) \rightarrow p \omega$ of $\boldsymbol{S}^{n-1} \times R$ onto $\boldsymbol{R}^{n}$ has an analog for $X$, namely the differentiable mapping $\phi:(k M, a) \rightarrow k a K$ of $(K / M) \times A$ onto $G / K$. Both maps are singular on certain lower dimensional sets. In contrast, the dual $\boldsymbol{E}\left(\boldsymbol{R}^{n}\right)$ of $\boldsymbol{R}^{n}$ (in the sense of $\S 3$ ) is doubly covered by $\boldsymbol{S}^{n-1} \times R$ and the dual $\Xi(G / K)$ of $G / K$ is diffeomorphic to $(K / M) \times A$ under the map $\hat{\phi}:(k M, a) \rightarrow k a M N$. This difference between the duals, $\boldsymbol{\Xi}\left(\boldsymbol{R}^{n}\right)$ 
and $\Xi(G / K)$, can be traced to the fact that whereas a hyperplane in $R^{n}$ is invariant under the geodesic symmetry with respect to a point in the hyperplane, the analogous statement does not hold for a horocycle in $X$.

Let $W$ be the Weyl group of $G / K$ acting on the Euclidean space $A$, let $S(A)$ be the symmetric algebra over $A$ and $I(A)$ the set of elements in $S(A)$, invariant under $W$.

The mapping $\phi$ above gives rise to an isomorphism $\Gamma$ of $D(X)$ onto $I(A)[15$, Theorem 1], [18, p. 432]; the mapping $\hat{\phi}$ also gives rise to an isomorphism $\hat{\Gamma}$ of $D(\Xi)$ onto $S(A)$. These isomorphisms can be described explicitly in Lie algebra terms. Concerning Problems A, B and $\mathrm{C}$ we have the following results [19], [22].

ThEOREM 6.1. The mapping $f \rightarrow \hat{f}$ is a linear one-to-one mapping of $D(X)$ into $D(\Xi)$. There is an isomorphism $D \rightarrow \hat{D}$ of $D(X)$ into $D(\Xi)$ such that

$$
(D f)^{\wedge}=\hat{D} \hat{f} \text { for } f \in D(X) .
$$

The isomorphism $D \rightarrow \hat{D}$ is defined as follows. The Iwasawa decomposition above corresponds to an ordering of the dual space of the Euclidean space $A$. In particular, the restricted roots of the symmetric space $X$ are ordered; as usual let $2 \rho$ denote the sum of the positive restricted roots, counted with multiplicity. Let $p \rightarrow p^{\prime}$ denote the automorphism of $S(A)$ given by $H^{\prime}=H+\rho(H)$ for $H \in A$. Then $\hat{D}=\hat{\Gamma}^{-1}\left(\Gamma(D)^{\prime}\right)$ for $D \in D(X)$.

Theorem 6.2. Suppose the Lie group $G$ is a complex Lie group. Then there exist operators $\square \in D(X), \Lambda \in D(\Xi)$ with the following property:

$$
\begin{aligned}
f & =\square\left((\hat{f})^{\curlyvee}\right), & & f \in D(X), \\
\int_{X}|f(x)|^{2} d x & =\int_{\Xi}|\Lambda \hat{f}(\xi)|^{2} d \xi, & & f \in D(X),
\end{aligned}
$$

where $d x$ and $d \xi$ are the G-invariant measures on $X$ and $\Xi$, suitably normalized.

We shall indicate the definition of $\square$ and $\Lambda$. Let $\pi$ denote the product of the positive restricted roots (without multiplicity). If $A$ is identified with its dual by means of the Killing form of the Lie algebra of $G$ then $\pi \in S(A)$ and $\pi^{2} \in I(A)$. Then, except for certain constant factors, $\Lambda$ equals $\hat{\Gamma}^{-1}\left(\pi^{\prime}\right)$ and $\square$ equals $\Gamma^{-1}\left(\pi^{2}\right)$. (Here $\pi^{\prime}$ is the image of $\pi$ under the automorphism $p \rightarrow p^{\prime}$ above.)

Theorem 6.2 holds more generally if $G$ has only one conjugacy class of Cartan subgroups, but the differential operators $\square$ and $\Lambda$ are 
then defined somewhat differently. For a general real $G$, the operators $\square$ and $\Lambda$ are integro-differential operators, and the analog of (2) is still valid. (See [22].)

Applications. It was mentioned earlier how the inversion formula (1) $\S 1$, to a certain extent, can reduce a partial differential equation with constant coefficients to an ordinary differential equation. Similarly, since $\square$ naturally commutes elementwise with $D(X)$, the inversion formula in Theorem 6.2 can reduce an invariant differential equation on $X$ to a differential equation on the Euclidean space $A$ (see [19]); this differential equation on $A$ is obtained via the mapping $\Gamma$, in particular it has constant coefficients, hence solvable.

In the case when $G$ is not complex better results are obtained by restricting the transform $f \rightarrow \hat{f}$ to radial functions $f$ on $X$, that is functions invariant under the action of $K$ on $X$. In fact it turns out that this transform converts each operator $D \in D(X)$ into a differential operator on $A$ with constant coefficients, resulting in the existence of a fundamental solution for $D$. The proofs of these results are based on Harish-Chandra's work on harmonic analysis on the group $G$ (see [20] and the references given there).

7. $p$-planes and $q$-planes in $R^{p+q+1}$. Let $p$ and $q$ be two integers $\geqq 0$ and put $n=p+q+1$. A $p$-plane $E_{p}$ in $R^{n}$ is by definition a translate of a $p$-dimensional vector subspace of $\boldsymbol{R}^{n}$. Let $G(p, n)$ denote the manifold of $p$-planes in $R^{n}$ and $G^{*}(p, n)$ the set of $p$-planes which do not pass through 0 . The projective duality between points and hyperplanes in $R^{n}$, realized by the polarity with respect to $S^{n-1}$ generalizes to a duality between $G^{*}(p, n)$ and $G^{*}(q, n)$. In fact, if $a \neq 0$ in $R^{n}$, let $E_{n-1}(a)$ denote the polar hyperplane. If $a$ runs through a $p$-plane $E_{p} \in G^{*}(p, n)$ then the hyperplanes $E_{n-1}(a)$ intersect in a unique $q$ plane $E_{q} \in G^{*}(q, n)$ and the mapping $E_{p} \rightarrow E_{q}$ is the stated duality.

We have now an example of the framework in $\$ 2$, although convergence difficulties make the results (Theorem 7.1) very restrictive. Let $X=G(p, n)$ and $G$ the group of rigid motions of $\boldsymbol{R}^{n}$, acting on $X$. Given a $q$-plane $E_{q}$ consider the family $\xi=\xi\left(E_{q}\right)$ of $p$-planes intersecting $E_{q}$. If $E_{q}^{\prime} \neq E_{q}^{\prime \prime}$ then $\xi\left(E_{q}^{\prime}\right) \neq \xi\left(E_{q}^{\prime \prime}\right)$; thus the set of all families $\xi$ - the dual space $\Xi$-can be identified with $G(q, n)$. In accordance with this identification, if $E_{p}=x \in X$ then $\check{x}=\check{x}\left(E_{p}\right)$ is the set of $q$ planes $E_{q}$ intersecting $x$.

The manifold $G(p, n)$ is a fibre bundle with base space $G_{p, n}$, the manifold of $p$-planes in $R^{n}$ through 0 , the projection $\pi$ of $G(p, n)$ on $G_{p, n}$ being the mapping which to any $p$-plane associates the parallel $p$-plane through 0 . Thus the fibre of this bundle $\left(G(p, n), G_{p, n}, \pi\right)$ is $\boldsymbol{R}^{n-p}$. If $f \in \mathcal{E}(G(p, n))$ let $f \mid F$ denote the restriction of $f$ to an arbi- 
trary fibre $F$ and let $\Delta_{F}$ denote the Laplacian on $F$. The linear transformation $\square_{p}$ of $\mathcal{E}(G(p, n))$ given by

$$
\left(\square_{p} f\right) \mid F=\Delta_{F}(f \mid F), \quad f \in \mathcal{E}(G(p, n)),
$$

for each fibre $F$, is a differential operator on $G(p, n)$. Because of convergence difficulties we do not define the measures $\mu$ and $\nu$ directly but define $\hat{f}$ by

$$
\hat{f}\left(E_{q}\right)=\int_{E_{q}}\left(\int_{a \in E_{p}} f\left(E_{p}\right) d \sigma_{p}\left(E_{p}\right)\right) d_{q}(a)
$$

whenever these integrals exist. Here $d \sigma_{p}$ is the invariant measure on the Grassmann manifold of $p$-planes through $a$ with total measure 1, $d_{q}$ is the Euclidean measure on $E_{q}$. The transform $g \rightarrow \check{g}$ is defined similarly (interchanging $p$ and $q$ ). For $p=0$ we get the situation in $\$ 3$. Let $\delta^{*}\left(R^{n}\right)$ be as in $\S 3$ and let $\varsigma^{*}(X)$ be the image of $\delta^{*}\left(R^{n}\right)$ under the operator $L_{p}: f \rightarrow \int_{E_{p}} f(a) d_{p}(a)\left(f \in \mathcal{S}^{*}\left(R^{n}\right)\right)$. Then the following result holds [21].

Theorem 7.1. The algebras $D(X)$ and $D(\Xi)$ are generated by $\square_{p}$ and $\square_{q}$, respectively. The mapping $f \rightarrow \hat{f}$ is a linear one-to-one mapping of $\mathcal{S}^{*}(X)$ onto $\mathcal{S}^{*}(\Xi)$ such that

$$
\left(\square_{p} f\right)^{\wedge}=\square_{q} \hat{f}
$$

If $n$ is odd then

$$
f=c\left(\square_{p}\right)^{(n-1) / 2}\left((\hat{f})^{2}\right), \quad \quad f \in \mathcal{S}^{*}(X),
$$

where $c$ is a constant, independent of $f$.

\section{BIBLIOGRAPHY}

1. W. A. Borovikov, Fundamental solutions of linear partial differential equations with constant coefficients, Trudy Moscov. Mat. Obšč. 8 (1959), 199-257.

2. R. Courant and A. Lax, Remarks on Cauchy's problem for hyperbolic partial differential equations with constant coefficients in several independent variables, Comm. Pure Appl. Math. 8 (1955), 497-502.

3. P. Funk, Über eine geometrische Anwendung der Abelschen Integralgleichung, Math. Ann. 77 (1916), 129-135.

4. L. Gårding, Transformation de Fourier des distributions homogènes, Bull. Soc. Math. France 89 (1961), 381-428.

5. I. M. Gelfand, Integral geometry and its relation to the theory of group representations, Uspehi Mat. Nauk. 15 (1960), 155-164=Russian Math. Surveys, 1960.

6. I. M. Gelfand and M. I. Graev, Analogue of the Plancherel formula for the classical groups, Trudy Moscov. Mat. Obšč. 4 (1955), 375-404.

7. - The geometry of homogeneous spaces, group representations in homogeneous spaces and questions in integral geometry related to them. I, Trudy Moscov. Mat. Obšč. 8 (1959), 321-390.

8. I. M. Gelfand, M. I. Graev and N. Vilenkin, Integral geometry and its relation to 
problems in the theory of group representations, Vol. 5, Generalized Functions, Fizmatgiz, Moscow, 1962.

9. I. M. Gelfand and M. A. Naimark, Unitary representations of the classical groups, Trudy Mat. Inst. Steklov. 36 (1950), 288.

10. I. M. Gelfand and S. J. Shapiro, Homogeneous functions and their applications, Uspehi Mat. Nauk 10 (1955), 3-70.

11. A. A. Hachaturov, Determination of measures in domains of an n-dimensional space by their values on all half spaces, Uspehi Mat. Nauk 9 (1954), 205-212.

12. Harish-Chandra, The Plancherel formula for complex semisimple Lie groups, Trans. Amer. Math. Soc. 76 (1954), 485-528.

13. - Fourier transforms on a semisimple Lie algebra. I, II, Amer. J. Math. 79 (1957), 193-257; 653-686. 760.

14. — , A formula for semisimple Lie groups, Amer. J. Math. 79 (1957), 733-

15. - Spherical functions on a semisimple Lie group. I, II, Amer. J. Math. 80 (1958), 241-310; 553-613.

16. S. Helgason, Differential operators on homogeneous spaces, Acta Math. 102 (1959), 239-299.

17. - Some remarks on the exponential mapping for an affine connection, Math. Scand. 9 (1961), 129-146.

18. - Differential geometry and symmetric spaces, Academic Press, New York, 1962.

19. - Duality and Radon transform for symmetric spaces, Amer. J. Math. 85 (1963), 667-692.

20. - - Fundamental solutions of invariant differential operators on symmetric spaces, Bull. Amer. Math. Soc. 69 (1963), 778-781.

21. - The Radon transform on Euclidean spaces, compact two-point homogeneous spaces and Grassmann manifolds (to appear).

22. - The Plancherel formula for the Radon transform on symmetric spaces (to appear).

23. F. John, Bestimmung einer Funktion aus ihren Integralen über gewisse Mannigfaltigkeiten, Math. Ann. 100 (1934), 488-520.

24. - - Plane waves and spherical means, applied to partial differential equations, Interscience, New York, 1955.

25. A. A. Kirillov, On a problem of I. M. Gelfand, Dokl. Akad. Nauk SSSR 137 (1961) = Soviet Math. Dokl. 2 (1961), 268-269.

26. J. Radon, Über die Bestimmung von Funktionen durch ihre Integralwerte längs gewisser Mannigfaltigkeiten, Ber. Verh. Sächs. Akad. Wiss. Leipzig Math.-Nat. Kl. 69 (1917), 262-277.

27. G. de Rham, Sur la reductibilité d'un espace de Riemann, Comment. Math. Helv. 26 (1952), 328-344.

28. L. Schwartz, Théorie des distributions. I, Hermann, Paris, 1950.

29. V. I. Semyanistyi, On some integral transforms in Euclidean space, Dokl. Akad. Nauk SSSR 134 (1960), 536-539=Soviet Math. Dokl. 1 (1960), 1114-1117.

30. - Homogeneous functions and some problems of integral geometry in spaces of constant curvature, Dokl. Akad. Nauk SSSR 136 (1961), 288-291=Soviet Math. Dokl. 2 (1961), 59-62.

31. H. C. Wang, Two-point homogeneous spaces, Ann. of Math. (2) 55 (1952), 177191.

Massachusetrs Institute of Technology 